Www.jmscr.igmpublication.org

Index Copernicus Value: 79.54

ISSN (e)-2347-176x ISSN (p) 2455-0450

crossref DOI: https://dx.doi.org/10.18535/jmscr/v7i4.168

\title{
Comparative Study of Sonographically Guided Catheter Drainage and Needle Aspiration in the Management of Liver Abscess
}

\author{
Authors \\ Dr Antony Jean', Dr Annitha Elavarasi Jayamohan ${ }^{2 *}$, Dr Ajit Kumar Reddy ${ }^{3}$, \\ Dr M.L. Prakash ${ }^{4}$ \\ ${ }^{1}$ Consultant Radiologist Aarthi Scans \\ ${ }^{2}$ Consultant Radiologist PD Hinduja Sindhi Hospital, \\ ${ }^{3}$ Assistant Professor, Dept. of Radiodiagnosis KIMS. Medical College, Bangalore \\ ${ }^{4}$ HOD, Dept. of Radiodiagnosis MGM, Medical College, Puducherry \\ *Corresponding Author
}

Dr Annitha Elavarasi Jayamohan

Consultant Radiologist PD Hinduja Sindhi Hospital

\begin{abstract}
Hepatic abscesses are a potentially lethal clinical entity; if prompt diagnosis and treatment are not accomplished, the condition is uniformly fatal.Over the years there has been significant improvement in the overall mortality rate of liver abscess which is mainly attributed to the introduction of effective antibiotic regimens, advances in imaging studies. A paradigm shift in the treatment modality of choice from the traditional open surgery to the minimally-invasive percutaneous drainagehas been observed. Various studies done in the recent past have failed to establish a concrete conclusion as to which of the two is better in the treatment of liver abscess. Hence the purpose of this study is to compare the above-mentioned treatment modalities and to identify the better option for treating patients suffering from liver abscess in our setup.

Keywords: Liver Abscess, Percutaneous drainage, Ultrasonography, Pig tail catheter, Aspiration, Image guided.
\end{abstract}

\section{Introduction}

Hepatic abscesses are a potentially lethal clinical entity; if prompt diagnosis and treatment are not accomplished, the condition is uniformly fatal.Literature reveals that the incidence of pyogenic liver abscess has remained similar over 60 years. More recent studies from the 1990s and 2000s have suggested small but significant increases in the incidence of pyogenic liver abscess (as high as 22 per 100,000 hospital admissions). In 1995, the World Health Organization estimated that 40 to 50 million people suffer from amoebic colitis or amoebic liver abscess worldwide, resulting in 40,000 to 100,000 deaths each year ${ }^{1}$. However, there has been significant improvement in the overall mortality rate of liver abscess as a whole ${ }^{2}$. This has been attributed to the introduction of effective antibiotic regimens, advances in imaging studies and critical care. There has also been a paradigm shift in the treatment modality of choice from the traditional open surgery to the minimallyinvasive percutaneous drainage ${ }^{5-8}$. The treatment of choice remains controversial. The spectrum of treatment options ranges from sole medical therapy to the more complex liver resection ${ }^{9-11}$. 
Nevertheless, the mainstay of treatment of liver abscess in the recent past has been either a percutaneous catheter drainage or percutaneous needle aspiration ${ }^{12-14}$. Various studies done in the recent past have not given a concrete conclusion as to which of the two is better in the treatment of liver abscess. Hence the purpose of this study is to compare the above-mentioned treatment modalities and identify the better option for treating patients suffering from liver abscess in our setup.

\section{Objectives}

1) To study the efficacy of Percutaneous catheter drainage (PCD) in Liver Abscess.

2) To evaluate the comparative effectiveness of percutaneous catheter drainage and percutaneous needle aspiration in liver abscesses.

\section{Material and Method}

This was a Prospective, randomized, open-label, blinded-endpoint (PROBE) study that evaluated the efficacy of Percutaneous Catheter Drainage over Aspiration in Amoebic and Pyogenic Liver Abscesses.

For our Study, Patients were selected from the Department of Medicine and Surgery of Mahatma Gandhi Medical College and Research Institute's Hospital. The Institutional Human Ethics Committee approval was obtained prior to the start of the study.

Study was conducted during the period from February 2011 to January 2012.

58 patients of liver abscess were included in the study, of which 8 were dropped during the course of the study for various reasons and lost to follow-up.

Patients of either gender above 18 years of age with radiologically imaged single or multiple liver abscess of above $5 \mathrm{cms}$ were included in the study.

Patients with previous drainage or aspiration history within one-year, hepatic malignancies, infected cysts of the liver, patients with already ruptured liver abscess, multiple small abscesses, multiple diffusely located abscesses and age below 12 years. were excluded from the study.
Patients fulfilling the inclusion/ exclusion criteria were included in the study, after obtaining full, voluntary informed consent. Detailed history of all patients was taken with thorough clinical examination and entered into a proforma during their stay and follow up. Routine investigations were done. After establishing diagnosis, medical treatment was initiated from day of admission.

Patients received either Cefetaxime $1 \mathrm{gm}$ IV BD or Ciprofloxacin $500 \quad \mathrm{mg}$ IV Intravenous metronidazole $100 \mathrm{ml}$ TID supportive therapy

On ascertaining response to therapy, patients were randomised into two groups using stacked envelopes with concealed randomisation sequence generated by STATWIN 2.3version into two groups: (1) for aspiration, (2) pigtail catheterization in addition to specific drugs. The two groups received the Interventions as described below. The response to therapy is assessed.

\section{Pre-procedure}

The Patients were restricted to NPO $4 \mathrm{hrs}$ prior to procedure. They were explained about the procedure in detail and were allowed to ask for clarifications if any, in presence of their family members and an impartial witness. An Informed Consent was taken for this study.

Skin Preparation was carried out and a Lidocaine test dose was administered. A Intravenous line was placed and bleeding parameters checked. Abscess drainage tray with (a) Pig tail Catheter $8.5 \mathrm{~F}$ to $12 \mathrm{~F}$ or (b) 18 Gauge Aspiration Needle as per the Study Group Assignment of the Patient were arranged for, along with local anesthesia and syringes prior to the start of the procedure. All procedures were done under strict aseptic precautions.

\section{Imaging technique}

USG abdomen was done, using longitudinal, oblique, transverse planes to visualize almost all part of liver. LOGIQ 5 Expert and LOGIQ 5 Pro from GE Healthcare with linear and curved transducers were used for all patients in this study to visualize Intercostal and sub-costal planes. 
Patients were positioned on the right, in supine followed by left lateral positions to gain adequate visibility of the imaged organs. Size, volume, location, content, echo pattern, vascularity both sagittal and longitudinal view. All the liver lesions suggestive of liver abscess were examined in detail. Doppler was used to evaluate the vascularity of the parenchyma surrounding the abscesses for determining optimal route for drainage free from vessels in the vicinity.

\section{Common precautions}

Coagulation factors were determined in all patients before the scheduled procedure to rule out any bleeding disorder. Three patients with evidence of coagulopathy received appropriate blood products for correction to acceptable levels of coagulation factors. Care was taken to ensure that the drainage route was free from interference and away from major blood vessels, intra-abdominal organs such as the gall bladder, stomach, bowel loops etc. Specific care was taken to avoid damage to the pleura by adopting the anterior or lateral approach, below the $6^{\text {th }}$ intercostal space in the mid clavicular or midaxillary line respectively.

For catheter drainage, we ensured that all the holes of the catheter were inside the cavity to prevent infection to the peritoneal space and organs.

The wall of the abscess on the surface of the Liver was ensured to be over 1.5 to $2.0 \mathrm{cms}$ to ensure its viability.

\section{Percutaneous drainage}

For Percutaneous catheter drainage (Figure 1), appropriately sized catheters ( 8 to 12 French pigtail catheter) were introduced into the abscess cavity as per Single Step Catheter Method (described below). The patients were positioned in supine, oblique or lateral positions depending upon the location of the abscess and their approachability with due consideration for the patient's comfort.

Careful localization of the abscess and proper selection of the entry site and trajectory were required. The site of entry of the needle or catheter was planned taking into consideration the size, shape, multiplicity and location of the abscesses. If the abscess was imaged to be long, the trajectory of the puncture was planned such that the inserted catheter lies throughout the length of the cavity. The approaches were planned to choose an entrance site at the upper or lower end of the cavity so that the catheter traverses the entire length or width. If the abscess was imaged to be elongated transversely, the catheters were placed in that transverse plane. In case of multiple septations in the abscess cavity, the trajectory was planned so that maximum cavities could be traversed.

Thus, the anatomical approach for the procedure is prepare and planned to facilitate unhindered access to the abscess. The optimal route of access traversed the least possible amount of liver tissue and avoided bowel and pleura. The catheter should be placed at the bottom of the abscess for optimal drainage. If needed, steps to shift the internal anatomy of the patient were undertaken to facilitate the passage of the catheter.

Pre-procedural anaesthesia was administered after checking the test dose site for reactions.

Inj. Lidocaine (drawn from fresh $20 \mathrm{ml}$ bottles) was used as the local anaesthesia of choice. In patients complaining of burning sensation during administration, Injectable Sodium Bicarbonate was added and administered along with the lidocaine to counteract the low $\mathrm{pH}$ as well as the pain.

A skin nick was given at the predetermined site with an 11-size surgical blade. Mosquito forceps was used to separate the superficial muscles and the prepared trocar catheter was introduced under USG guidance. Once we confirmed that the catheter needle tip to be well within the abscess, the needle was slowly withdrawn. The trocar was placed in position, and a $10 \mathrm{ml}$ Syringe was used to aspirate the contents of the abscess for confirmation of the abscess. The trocar was left in situ and the catheter was further introduced into the cavity.

In patients with multiple abscesses, each abscess was drained with a separate catheter: two catheters were used in four patients and one patient needed three catheters. The catheters were connected to a completely closed collecting system and routine 
catheter care was instituted. Aspiration was performed with the catheter until no more pus was removed. The catheter then was secured to the skin for continuous external drainage and was left in place until production of content stopped. Residual cavities of abscesses were managed by catheter repositioning and aspiration. A daily estimate of the amount, colour, and consistency of the drainage fluid was recorded. Irrigation of the catheter with sterile saline was done daily to avoid catheter blockage. Catheters were removed when the patients showed clinical improvement (i.e..defervescence and relief from local symptoms and normalization of elevated leukocyte counts), the catheter output dropped to less than $10 \mathrm{ml} / 24 \mathrm{hr}$ for 2 consecutive days, and follow-upsonography showed negligible residual cavity. Aspirated pus was examined and microbiologic tests were performed to determine the causative organism. Broad spectrum antibiotics were initiated. Once the laboratory results were available, antibiotics were changed on the basis of sensitivity tests. However, patients in whom pus culture findings were negative were continued on the same broad spectrum antibiotics and anti-amoebic drugs. The antibiotics and metronidazole were given for duration of 10 days and 14 days, respectively.

All patients were followed up to assess the time needed for clinical improvement. Length of hospital stay and development of any complications. Periodicsonography was done every third day ideally (4 or $5^{\text {th }}$ days in some instances) to assess the cavity size until the patients were hospitalized. Patients in the percutaneous needle aspiration group who did not improve clinically after the first aspiration and continued to have leukocytosis or showed refilling of the abscess cavity on followupsonography were subjected to a second aspiration. Failure of the patient to improve after a second aspiration was considered as failure of aspiration therapy. After discharge, all patients were followed up with periodic clinical and sonographic examinations to assess for any recurrence of the disease and to monitor the size of the abscess cavity. The patients were examined weekly for I month, monthly for the next 3 months. and at two monthly intervals thereafter until complete resolution of the abscess was achieved. Treatment was considered successful if all of the following criteria were met: The patients improved clinically (i.e. subsidence of fever and local signs and symptoms). Elevated leukocyte counts were normalized, follow-up imaging showed resolution of the abscess (total resolution or reduction in size to $<3 \mathrm{~cm}$ ), and no evidence of relapse or recurrence was seen during follow-up.

\section{Catheter care}

Daily irrigation of catheter once or twice with sterile normal saline was done, which prevents the blockage of catheter, still if catheter is blocked stylette was reintroduced to clean the catheter. Daily estimation of volume,colour and consistency of the drainage fluid was recorded.

In few cases hyoluronidase was injected in to the cavity through the catheter where pus was found to be very thick.

Catheter was kept in site for an average period of 13 days. The duration varied in individual cases depending on the quantity of pus, or presence of biliary fistula. Follow up was done using ultrasonography to note the shrinkage in size of the cavity every $4^{\text {th }}$ or $5^{\text {th }}$ day.

\section{Needle aspiration}

In the patients assigned to the needle aspiration group, evacuation of pus from an abscess was performed with an 18-gauge disposable trocar needle (Figure 2).

The patients were positioned in supine position, after pre-procedural workup. Ultrasound examination of the liver is done to locate the abscesses and plan an approach. The site for needle insertion is marked and prepared by cleaning with betadine and spirit solutions. After draping the site, lignocaine based local anaesthesia was administered in the same trajectory of the needle insertion.

The Aspiration procedure was divided into four individual steps. (1) Localization of the needle tip in the lesion, (2) application of suction, (3) movement of the needle, and (4) removal of the needle. 
Localization of the needle tip in the site of the mass is crucial and likely the most important step in our procedure and was done meticulously under close ultrasound guidance - The principle is to ensure that the tip should be near the bottom of the abscess. For application of suction, a two-person technique was used with one person applying suction and the second holding and moving the needle advanced into the abscess cavity and the contents aspirated with negative pressure in an attempt to completely evacuate the cavity. A $20 \mathrm{ml}$ syringe was used to apply suction and contain the evacuated contents. Two syringes were used, one after the other. The aspirated evacuated contents of the syringes were emptied into a container for visual and volumetric study and after a sample was sent for culture analysis, the rest were discarded.

Aspiration is done till no fluid could be evacuated with moderate suction. Ultrasound is used to determine remnant fluid level of the abscesses. Once it was established by ultrasound as well as by aspiration attempt that no more fluid was available for aspiration, the procedure was concluded. Lavage with saline is done for abscesses with residual abscess contents and aspirated till complete evacuation.

Patients were reviewed every 3 days, and the size of the abscess cavity was recorded. If there was no significant reduction in the abscess cavity on control examination, aspiration was repeated. Lack of response to the second aspiration attempt was considered failure of treatment, and a catheter for continuous drainage was introduced. Patients who needed this treatment were not included in the PCD group.

All patients underwent clinical follow-up and monitoring during daily rounds until they were discharged from the hospital. Follow-up sonography was performed 24 hours after intervention and repeated every 3 days, and the size of the abscess was recorded. Criteria for successful treatment were clinical subsidence of infection and sonographic evidence of abscess resolution, such as disappearance or marked decrease in the abscess cavity (more than $50 \%$ reduction of longest diameter be-fore treatment)

After discharge from the hospital, patients underwent follow-up evaluations in the outpatient clinic at least once a week during treatment and biweekly until 6 months from the beginning of the treatment. Patients discharged with a catheter underwent follow-up sonography every 3 days until there was no catheter output for 24 hours, and then the catheter was removed. Patient outcome, including length of hospital stay, complications related to the procedure, and treatment failure, were recorded.

\section{Results}

PCD was successful in $15(60 \%)$ of the 25 patients, excluding 4 lost to follow up whose data was not considered for PROBE analysis. Eleven patients responded to a single aspiration. The other 14 patients were subjected to a second aspiration 27 days (average, 3 days) later for one or both of the following reasons: persistence of fever and leukocytosis $(n=11)$ and reaccumulation of the abscess as shown by follow-upsonography $(n=I 2)$. Only four of these 14 patients responded to a second aspiration. Percutaneous needle aspiration was considered unsuccessful in 10 patients (40\%) because they failed to improve clinically or radiologicallyeven after a second aspiration. Rapidreaccumulation in the abscess cavity was seen in eight of these 10 patients within 36 days after the second aspiration. The average volume of abscesses $(325 \mathrm{ml})$ in the 10 patients in whom needle aspiration was unsuccessful was significantly ( $p$ $<.05)$ larger than the average volume $(178 \mathrm{ml})$ of the abscesses in the 15 patients who responded to one or two aspirations. However, other patient and abscess characteristics were similar in these two groups of patients. Subsequently, all of these 10 patients were successfully treated with percutaneous catheter drainage (however, in our study, these patients are not included in the PROBE analysis of the catheter drainage group). CD was successful in all 29 patients $(100 \%)$ of the percutaneous catheter drainage group. In most patients $(n=26)$, duration of catheter drainage varied from 3 to 15 days 
(average, 7 days). The duration was prolonged (35 days) in only one patient. This patient and three more patients were discharged with catheters in situ when they became clinically stable but had persistent drainage from the catheter; the patients attended the outpatient department on alternate days until catheter removal. Among the successfully treated patients, the average time needed for clinical relief (defervescence) and mean hospital stays were similar in the two treatment groups. Although the time needed for reduction of the abscess cavity to $50 \%$ of its original size was significantly more in the needle aspiration group than in the percutaneous catheter drainage group (11 days versus 5 days), the average time taken for total or near total resolution of the abscess was similar in both groups of patients. In three patients, small $(2-3 \mathrm{~cm})$ residual cavities were still present at 10 to12 weeks after drainage; these patients were lost to further followup. No relapse was documented on clinical and sonographic examination during follow-up (range, 37 weeks; mean, 20 weeks).

\section{Complications}

No major complications were encountered. Three patients, two in the catheter group and one in the aspiration group, had minor complications. One patient complained of severe pain at the catheter entry site that was relieved with oral analgesic. A pericatheter leak developed in another patient because of blockage of the catheter with debris; the leak stopped after flushing of the catheter with sterile saline. Haemorrhage within the abscess cavity developed in the third patient during needle aspiration, as suggested by the sudden appearance of echogenic foci within the homogeneously hypoechoic abscess cavity and the mixture of blood with the aspirated material; this patient was treated conservatively with bed rest and IV fluids.

The age group in this study ranges from the 25 years - 70 years. Highest incidence of age is found between $3^{\text {rd }}-6^{\text {th }}$ decades. Other studies showed incidence of $75-90 \%$ in $2-5^{\text {th }}$ decades. Our study shows $70.7 \%$ in the age group of $3^{\text {rd }}-6^{\text {th }}$ decades. Youngest one is 25 years old male and oldest 70.In this study group 56 cases were male and 2 cases are female. Male predominance is reported always in literatures. Sex incidence, ratio being, Male: Female 13.5: 1. Liver abscess is commonly seen in low socio economic group. In our study almost all patients were from low socio economic group. 55 patients belongs to low, remaining belonged to mid income group. Ochsner and De Bakey attribute higher incidence of alcoholism in males, which predisposes hepatitis and trauma. Very few workers have tried to confirm actual relationship of alcohol to liver abscess but exact pathology is not known. According to some Authors the incidence varies from $20 \%-62.5 \%$. In our series it is at $88 \%$ and it is more common in persons who consumed alcohol more than 40 years.

Among 58 patients, 10 patients gave past history of intestinal amoebiasis, concomitant history of diarrhea present in 18 cases. A previous history /attack of amoebic dysentery as a rule antedate the onset of ALA by days, weeks, month or year. There are cases in which history of amoebiasis may not even be traced and the subject may be carrier.

In our study of 58 cases of liver abscess, 57 cases gave history of right upper quadrant pain, which was dull aching, and a few patients gave history of pain referring to right shoulder. Fever with chills and rigors seen in 47 cases, weight loss was complained in $42(72 \%)$ cases, anorexia in $27(46.5 \%)$ cases. 18 cases gave history of diarrhoea and 15 patients had history of chest pain in association with dyspnoea, cough in 17 cases, in most cases cough was dry in nature. Very few were known cases of COPD has cough with expectoration and 7 patients has history of dyspnoea.

$40 \%$ of cases subjected to a Chest X-ray had elevated or right dome of the diaphragm with restricted movements.

The elevated right dome of the diaphragm was due to upper enlargement of liver, which occurs, in liver abscess. $12 \%$ of the cases had pleural effusion, basal lobe consolidation seen in 9 cases. Cardiomegaly and involvement of pericardium was not seen in any of the cases. COPD and chronic bronchitis in chest $\mathrm{x}$-ray was seen in most of middle-aged male patients. 


\section{JMSCR Vol||07||Issue||04||Page 1016-1026||April}

Air fluid level seen below right diaphragm in 2 cases, probably due to gas forming organisms.

USG is a very important tool, both in diagnosis and therapeutic management of liver abscess. It is noninvasive, 80-90\% accurate, capableof delineating liver lesions as small as $2 \mathrm{cms}$ in diameter.The long axis measurement of abscesses varied from 5 to $15 \mathrm{cms}$ with a mean of $8.3 \mathrm{cms}$. The smallest abscess was $62.5 \mathrm{cc}$ and the largest measured $1687 \mathrm{cc}$.

\section{Figure 1}

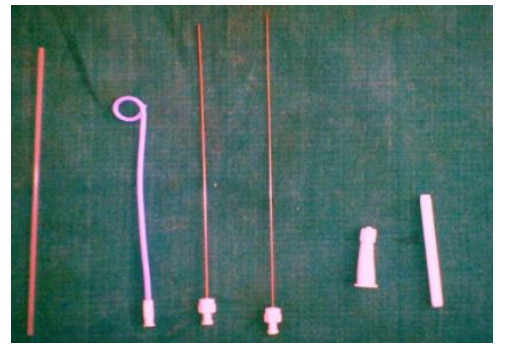

a) PCD tray

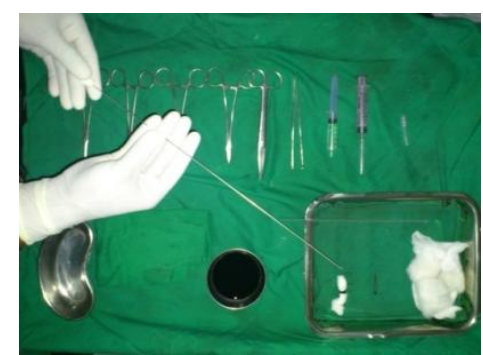

b) Trochar with catheter

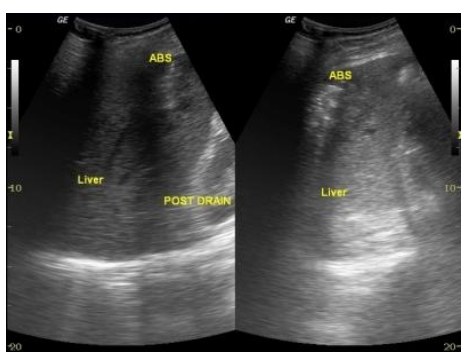

c) Pre and post PCD

Figure 2

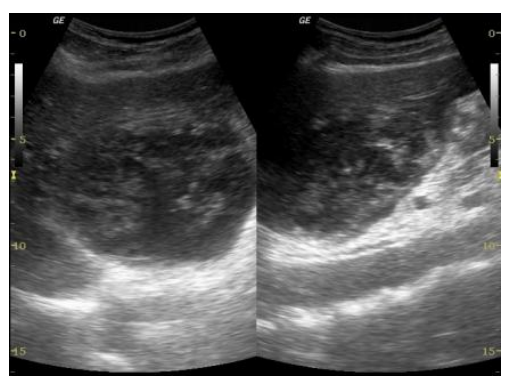

a) Abscess in transverse and longitudinal view

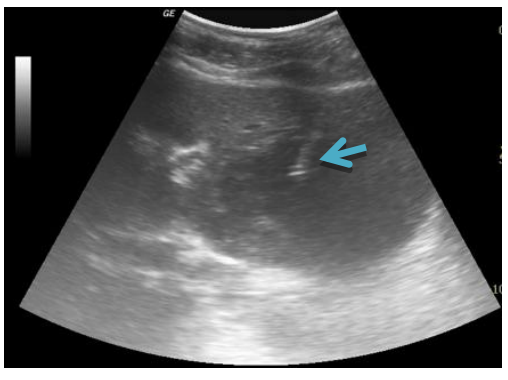

b) Needle within abscess

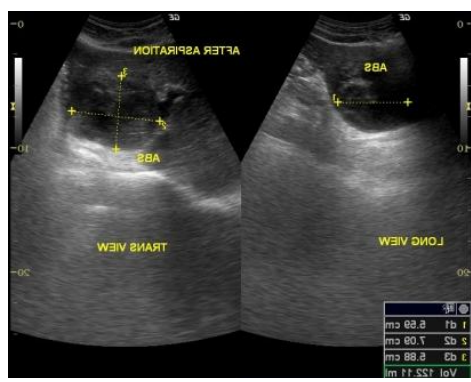

c) Post aspiration.

\section{Discussion}

Pain and fever were the most common symptomsinour study, comparable with the study by Gerzof, et al. $(n=12)$. The abscess characteristics like site of abscess, location of abscess, no of abscesses was comparable with the same study and other standard studies ${ }^{15-20}$. Etiology of the abscess was not comparable with western studies, because incidence of amoebic abscess in tropical countries like ours is more ${ }^{21-23}$. In pyogenic abscess most common cause was pyelophlebitic route ${ }^{24-27} ; 5$ out of 9 cases had evidence of peptic ulcer, while most common cause in western series is biliary ${ }^{28-31}$, excluding cryptogenic ${ }^{32-26}$.

\section{Success rates}

Relief of symptoms and sonographic evidence of collapse of cavity were considered as the criteria for success of the study irrespective of the mode of intervention. In our study success rate of $96.5 \%$ was seen comparable with Rajak, et al. ${ }^{31}$ (1998) and Eric von Sonnenberg ${ }^{37}(1985)$ with $100 \%$ success rate. K.P.Wong ${ }^{41}$ with success rate of $85 \%$, and Gerzof, et al. ${ }^{42}$ with $83 \%$ success rate.

\section{No of cavity}

Most of the abscesses are solitary; however multiple abscesses are not usual. In the present study, 39 
cases $(67.2 \%)$ had solitary abscess including 7 cases of left lobe abscess. 19 (32.8\%) cases showed multiple abscesses.

In the past few years, imaging guided percutaneous treatment (needle aspiration or catheter drainage) has replaced surgical intervention as the principle treatment for liver abscesses. Among the many advantages of needle aspiration over catheter drainage include the following: Needle aspiration is less invasive and cheaper in the Indian context; follow-up catheter care can be avoided, requiring lesser medical or nursing care; and multiple cavities can be aspirated in a single session and Patients can be discharged earlier, with lesser impact to their out-of-work burden. However, as our study shows, needle aspiration, if limited to two attempts, has a significantly lower success rate than catheter drainage $(60 \%$ versus $100 \%)$. The success rate of percutaneous needle aspiration in the various series reported in the literature varies from $79 \%$ to $100 \%$. The relatively lower success rate $(60 \%)$ of percutaneous needle aspiration in our study is possibly related to the fact that repeated aspiration was attempted only once in cases of nonresponsive initial aspiration; this procedure is in contrast to that in most of the other studies, in which repeated aspirations were done up to three or four times.

In our study also, the success rate after one aspiration was only $44 \%$; it increased to $60 \%$ after two aspirations. A higher success rate would likely have been achieved if multiple repeated aspirations were attempted. However, subjecting the patients to multiple needle aspirations (with the average number of aspirations per patient ranging from 1.4 to five) over a short period varying from 5 to 14 days is a traumatic and unpleasant experience for the patients and may not be acceptable to many. Moreover, even multiple attempts do not guarantee a $100 \%$ cure rate. For these reasons, we preferred to subject the patients to percutaneous catheter drainage after failure by second aspiration. The average size of the abscess in our study was larger than in other series. In contrast to some of the earlier reports that show that the initial size of the abscess cavity did not affect the ultimate outcome, we believe that large abscesses are more difficult to evacuate completely in one attempt, necessitating repeated aspirations. The average volume of abscesses in the 10 patients in whom percutaneous needle aspiration was unsuccessful was significantly larger than the average volume of the abscesses in the 15 patients who responded to one or two aspirations. One important reason for failure of needle aspiration is the inability to completely evacuate the thick viscous pus that may be present in some of the abscesses; this pus was seen in three of our patients. Rapid reaccumulation of the abscess after needle aspiration is another problem, described by Dietrick and seen in 12 of our patients after first aspiration and eight patients after second aspiration. In some of the patients (two in our series), this rapid refilling could have been due to biliary communication; however, in most patients no obvious predisposing factor existed, and the continuing inflammatory process itself probably contributed to the re-accumulation of fluid.

In contrast to percutaneous needle aspiration, percutaneous placement of an indwelling catheter provides continuous drainage; hence, the problems of incomplete evacuation and reaccumulation are not associated with catheter drainage, accounting for the high success rates of catheter drainage reported in most of the earlier studies and also seen in our series. The only reasons for failure of percutaneous catheter drainage, as reported in some of the earlier series, have been either thick pus not amenable to percutaneous drainage (this problem can be overcome by using larger bore catheters) or premature removal of the drainage catheter (strict adherence to the criteria for catheter removal can prevent this problem). No recurrence occurred in any of our cases during the followup period. Our study suggests no meaningful difference in either the time taken for defervescence or the duration of hospitalization among the patients successfully treated with either technique. In keeping with the findings of earlier reports, both treatment techniques resulted in rapid clinical relief, with most patients showing resolution of fever, local symptoms, and leukocytosis within 3 days of the procedure. 
The average hospital stay (13 days) of the patients who underwent percutaneous catheter drainage in our study was shorter than that reported in two earlier series. One possible explanation is that all the patients in those series had pyogenic liver abscesses and continued to be hospitalized for the definitive treatment of the predisposing conditions such as diverticulitis, gall stones, gall bladder carcinoma, and so forth even after removal of catheter and resolution of the abscess. No such predisposing conditions were recognized in the small number $(\mathrm{n}=11)$ of patients with proven pyogenic liver abscesses encountered in our study. The shorter hospital stay could also be related to the fact that, unlike the practice in previous studies, we did not wait for total radiologic resolution of abscess cavity before discharge; the average volume of abscess at the time of discharge was $70 \mathrm{ml}$. Also, four of our patients were discharged with catheters in situ when they became clinically stable but had persistent drainage from the catheter. The time required for complete sonographic resolution of abscess cavities after percutaneous treatment ranges from 2 weeks to 9 months. In fact, total resolution may not occur, and small residual cavities may persist for years. Such cavities are usually indistinguishable from simple hepatic cysts. In a series of 30 patients, Singh and Kashyap noted much faster and more complete resolution of abscess cavity after percutaneous catheter drainage than after percutaneous needle aspiration. However, the results of our study suggest that although collapse of the abscess cavity is achieved earlier in patients undergoing catheter drainage than in those undergoing needle aspirations, the time needed for total resolution of the abscesses is similar in the two groups. Complications have been reported with both catheter drainage $(12 \%$ in the series of Lambiase et al.) and needle aspiration (4\% in the series of Baek et al.). Baek et al. and Giorgio et al. describe the much lower incidence of complications with percutaneous needle aspiration than with catheter drainage as one of the major advantages of needle aspiration. However, our study and some recent reports suggest that both procedures, if properly performed, are essentially safe procedures with minimal complications. Singh and Kashyap reported a $15 \%$ incidence of secondary bacterial contamination after multiple needle aspirations; however, other workers have not encountered this problem. Although secondary bacterial infection remains a distinct theoretic possibility with an indwelling catheter, this complication has been rarely reported in liver abscesses.

\section{Limitation}

One limitation of our study is that the patients included formed a heterogeneous group with abscesses of both amoebic and pyogenic causes existing in both groups. Also, many abscesses ( $\mathrm{n}=$ 19) were of indeterminate cause. Because our institution is a referral hospital, many of these patients had been partially treated with antibiotics and antiamoebic drugs before being referred to us; this probably accounts for the high percentage of abscesses with negative findings on pus cultures in our study. Some previous researchers have also had this experience. Hence, analysis of these cases does not allow prediction of a successful outcome based on the cause of abscesses. This information could be obtained from a prospective study that included a larger number of abscesses of a single cause.

\section{Conclusions}

In conclusion, although both percutaneous needle aspiration and catheter drainage are safe methods for the nonsurgical treatment of liver abscesses, catheter drainage is more effective than needle aspiration, which, if limited to two attempts, is associated with a high failure rate, especially in large abscesses and in abscesses with thick viscous pus. However, the results also indicate that needle aspiration with appropriate antimicrobial therapy will, in many patients, result in a cure. Also, among the successfully treated patients, no significant difference exists in the time required for clinical improvement, the duration of hospitalization, and the time needed for resolution of the abscess cavity in the two treatment groups. Unfortunately, our data do not allow us to predict the type of abscesses 
likely to respond to needle aspiration alone. Further prospective, randomized studies investigating needle aspiration alone are therefore warranted to specifically address this problem

\section{Acknowledgement}

The heading of the Acknowledgment section and the References section must not be numbered.

\section{References}

1. Michael J.Zinner, Seymour I. Schwartz, Harold ellis, Maingot's Abdominal Operations, $10^{\text {th }}$ edition, vol 2, Appleton and Lange, Chapter51: 1513-1545

2. Pope I M, ThomasP G, Pyogenic liver abscess, Amoebiasis and biliary Infection, Surgery of Liver and biliary tract, TH Glumgart, Churchill living stone, 1974; Vol -II 1135-1157.

3. Hoffner RJ, Kilaghbian T, Esekogwu VI, Hendevson SO. Common presentations of amoebic liver abscess. Annals of Emergency Medicine1999; 34 (3): 351-5.

4. Katzenstein D, Rickerson V, Braude Abraham : New concepts of amoebic liver abscess derived from hepatic imaging, serodiagnosis, and hepatic enzymes in 67 consecutive cases in San Diego. Medicine1982; 61: 237-46.

5. Lee KC, Yamazaki O, Hamba $\mathrm{H}$ et al. Analysis of 69 patients with amoebic liver abscess. Journal of Gastroenterology1996; 31 (1): 40-5.

6. Ochsner A, DeBackey M, Murray S pyogenic abscess of liver. An analysis of 47 cases with a review of literature. Am Jsurg, 1938; 40:292-319.

7. Charles F. Brunicardi, Dana K. Andersen, Thimothy R. Billiar, Schwartz's Principles of Surgery, $8^{\text {th }}$ edition, McGrawhill, 2005:1162-1164

8. Bismuth H. Surgical anatomy and anatomical surgery of the liver. In: Blumgart LH, editor . Surgery of the liver and biliary tract . Edinburgh (UK):

Churchill Livingstone; 1994.

9. McClusky DA 3rd, Skandalakis LJ, Colborn GL, Skandalakis JE. Hepatic surgery and hepatic surgical anatomy: historical partners in progress. World JSurg 1997;21:330-42.

10. Abdalla EK, V authey JN, Couinaud C. The caudate lobe of the liver. Implications of embryology and anatomy for surgery. SurgOncolClin N Am 2002;11:835-48.

11. Couinaud C.Liver anatomy: portal (and suprahepatic) or biliary segmentation. Digest Surg 1999;16:459-67.

12. Bismuth H. Surgical anatomy and anatomical surgery of the liver . World J Surg 1982;6:3-9.

13. Bismuth $H$. A text and atlas of liver ultrasound. London: Chapman and Hall Medical ; 1991. p. 2-15.

14. Soyer P. Segmental anatomy of the li ver: utility of a nomenclature accepted worldwide. AJR 1993;161:572-3.

15. Whitmore I. Terminologiaanatomica: new terminology for the new anatomist. Anatom Rec (New Anat) 1999;257:50-3.

16. Terminologiaanatomica: international anatomical terminol o-gy . FCA T. Thieme, Stuttgart, New York; 1998. p. 54-6.

17. Strasberg SM for the IHPBA Terminology Committee Survey. Terminology of hepatic anatomy and resections. HPB 1999;1:192201.

18. Courtney M. Townsend, Jr, R. Danielbeauchamp, B.Mark Evers, Kenneth I. Mottox, Sabiston Text Book of Surgery, $17^{\text {th }} \quad$ edition vol 2, Elsevier,2004;1534-1542

19. Marberg K, Czerniak P. Observations of isotope hepatoscanning in diagnosis and treatment of amoebic liver infection. Ann Intern Med1964; 60: 66.

20. Raus PW, Colleti PM, Quinn MF et al. Sonographic findings in hepatic amoebic abscess. Radiology1982; 145: 123-6. 
21. Sachdev GK, Dhol P. Colonic involvement in patients with amebic liver abscess : Endoscopic findings. Gastro-intestinal Endoscopy1997; 46 (1): 37-9.

22. Hanna RM, Dahniya MH, Badr SS, El Betagy A. Percutaneous catheter drainage in drug-resistant amoebic liver abscess. Tropical Medicine and International Health2000; 5 (8): 578-81.

23. Jain A, Kar P. HbsAg carrier with simultaneous amoebic liver abscess and acute hepatitis E. Indian J Gastroenterol 1999; 18 (3): 124.

24. Lamont N, Pooler NP. Hepatic amoebiasis. A study of 250 cases. QJ Med New Ser1958; 27: 319-412.

25. Chummy S. Sinnatamby, Last's Anatomy Regional and Applied, $10^{\text {th }}$ edition chapter5: 253-257

26. Petrs RS, Gitlin N, Libke RD, Amoebic Liver Diseases, Ann Rev med; 1982; 32: 161-74.

27. Sheila Sherlock, Disease of Liver and biliary system, 1991; $9^{\text {th }}$ Edition: 471477.

28. Sharma MP, Dasarathy S. et al, Variants of Amoebic liver abscess. Arch Med Res 1997; 28: 5272-73.

29. Theron P, Surgical Aspects of Amoebiasis, 1974; British Medical Journal 26: 123-126.

30. Subramaniam R. Krishnan K.T et al, J. Ass Phys Ind. 1970; 18: 729.

31. Ramachandran, S. Sivalingam R. S et al, $J$ trop Med Hyg 1972; 75: 23.

32. Data DV, Saha S, Singh SA et al, The Clinical Pattern and Prognosis of Patients with Amoebic Liver Abscess and Jaundice, Am J Digestive Disease 1973; 18: 883-98.

33. Turill FL, Brunham JR, Hepatic Amoebiasis, Am J Surgery 1996; 11: 42430.

34. Sharma MP, Verma N, Acharya SK, Clinical Profile of Multiple Liver Abscess, $J$. Association of Physicians, 1990; 38: 837-9.

35. P Kapoor, Joshi R, Multpli Amoebic Liver Abscess, A study of 56 cases, J. Top Med Hyg 1992; 75: 4-6.
36. Sharma MP, Sarin S K, Inferior Caval obstruction due to Amoebic Liver Abscess, $J$. Assoc Physicians India, 1990; 30: 243

37. Gregory $C$ et al, Ruptured Amoebic Liver Abscess, Archives of Surgery, 1985; 120:555-61.

38. R. Ramachandra et al, Syndrome of Amoebic Liver Abscess, British Journal of Surgery, 1976; 63: 220-225.

39. Jose. E. Thompson, R. Susan, Fortenza et al Amoebic Liver Abscess and Therapeutic approach, Rev Infectious Disease 1985; 7: 171-179

40. Lamaunt NM and Pooler NR, Hapetic Amoebiasis a study of 250 cases Quarterly Journal of Medicine, New Series 27, 1958; 107: 389-412.

41. David Faegenburg et al, Toxic Megacolon in Amoebic Colitis, 1967; Vol 1: 